\title{
Endokrine und mesenchymale Tumoren des Gastrointestinaltrakts
}

\author{
Steffen Leinung ${ }^{\mathrm{a}} \quad$ Joachim Mössner ${ }^{\mathrm{b}} \quad$ Johann Hauss $^{\mathrm{a}} \quad$ Jakob R. Izbickic $^{\mathrm{c}}$ \\ ${ }^{a}$ Klinik für Viszeral-, Thorax- , Transplantations- und Gefäßchirurgie, \\ ${ }^{b}$ Gastroenterologie/Hepatologie, Medizinische Klinik II, Universität Leipzig, \\ ${ }^{c}$ Abteilung für Allgemeinchirurgie, Chirurgische Klinik, Universitätsklinikum Hamburg-Eppendorf, Deutschland
}

Im vorliegenden Themenheft werden zwei Tumorentitäten interdisziplinär besprochen, die in Klinik, Diagnostik und Therapie sehr unterschiedlich sind. Beiden gemeinsam ist jedoch ihre Seltenheit.

Mit dem charakterisierenden Nachweis der Expression des c-kit-Proteins (CD117) wurde in den 1990er Jahren ein diagnostisches und therapeutisches Konzept für gastrointestinale Stromatumoren (GIST) entwickelt [1, 2], und es etablierte sich der Begriff GIST für die jetzt einheitlich zusammengefassten Tumoren. Zur gleichen Zeit wurde der Karzinoidbegriff zugunsten der Bezeichnung «neuroendokrine Tumoren» (NET) verlassen.

GIST treten als relativ homogene Tumorentität in Klinik, Diagnostik und Therapie in Erscheinung. Die GIST-Diagnose erfolgt häufig als Zufallsdiagnose im Rahmen einer akuten Blutung bzw. eines abdominellen Schmerz- oder Passageproblems. Durch die Einführung von Imatinib wurde ein effektives einheitliches Behandlungsregime entwickelt [3]. GIST gelten als potentiell maligne, so dass immer eine komplette Resektion Therapieziel ist. Durch die Tumorgröße und die multifaktorielle Operationsindikation aus Schmerz, Blutung, Passsageproblem und potentieller Malignität ist dem betroffenen Patienten die Operationsindikation beim wahrscheinlichen GIST plausibel zu vermitteln. Für den Operateur ist die chirurgische Therapie - überwiegend durch eine Hohlorganteilresektion - sicher und schnell zu realisieren.

Eine besondere Erfahrung und Infrastruktur sind die Grundvoraussetzungen für die Behandlung von weit fortgeschrittenen, multilokulären sowie metastasierten Tumoren [4].

Erbliche Faktoren haben nach bisherigem Kenntnisstand bei mesenchymalen gastrointestinalen Tumoren - abgesehen von der Häufung von Leiomyosarkomen im Rahmen der CarneyTriade - eine geringere klinische Bedeutung als bei NET.

Endokrine Tumoren verlangen sowohl vom Diagnostiker als auch vom Therapeuten eine überdurchschnittlich hohe Erfah- rung. NET stellen oft eine besondere Herausforderung an den Hausarzt dar, der bei der häufig unspezifischen, gelegentlich subklinischen Symptomatologie die Indikation zur Überweisung an einen Endokrinologen stellen muss. Auch für den hoch spezialisierten Internisten ist die NET-Diagnostik oft deutlich differenzierter und kostenintensiver als die GIST-Diagnostik, was diese Patienten in der Regel an universitäre Einrichtungen bindet. Aufgrund der verschiedenen Hormonaktivitäten gibt es im Vergleich zum GIST eine Fülle von Therapieoptionen [5, 6]. Gastrointestinale NET sind häufiger aufgrund der Auswirkungen der Hormonproduktion problematisch als wegen ihres generellen Malignitätspotentials. Die besonderen Anforderungen an die chirurgische Kompetenz bei der chirurgischen NET-Therapie resultieren daraus, dass die Lokalisation dieser Tumoren präoperativ häufig nicht vollständig bekannt ist, die Tumoren klein sind und sich zum Teil als multiple Tumoren präsentieren [7, 8]. Vorraussetzungen für die erfolgreiche chirurgische Lösung des Problems sind bei NET die intraoperative Organmobilisation mit einer bimanuellen Palpation durch einen erfahrenen Viszeralchirurgen, der intraoperative Ultraschall, die intraoperative Diaphanie, individuelle Operationstechniken, die Möglichkeit intraoperativer Insulin-Bestimmungen sowie Möglichkeiten zum histopathologischen Schnellschnitt. Wegen der Sensibilität und anatomischen Komplexität des Pankreas erfordert die NETChirurgie in diesem Bereich besonders hohe Fertigkeiten und eine große Erfahrung des Operateurs.

Im Rahmen des MEN-, des Cowden- und des Von-HippelLindau-Syndroms spielen familiäre Erkrankungen eine Rolle und führen bei ersterem auch zu prophylaktischen Operationen.

Eine besonders hohe Verantwortung bei der Dignitätseinschätzung dieser Tumoren hat der Pathologe, da diese Einschätzung nicht nur für die Folgetherapie- und Lebensplanung der Patienten eine Rolle spielt, sondern oft auch intraoperativ

\begin{tabular}{|c|c|}
\hline KARGER & (c) 2007 S. Karger GmbH, Freiburg \\
\hline $\begin{array}{l}\text { Fax +4976145207 } 14 \\
\text { E-mail Information@Karger.de } \\
\text { www.karger.com }\end{array}$ & $\begin{array}{l}\text { Accessible online at: } \\
\text { www.karger.com/cga }\end{array}$ \\
\hline
\end{tabular}


vom Chirurgen intensiv zu Rate gezogen wird, um die angemessene Eingriffsgröße mit dem entsprechenden Risiko im Einzelfall zu festzulegen. Wir wissen, dass uns in Hinblick auf den Tumor - trotz moderner Diagnostik - oft nur prognostische Hinweise zur Eingriffsplanung zur Verfügung stehen und erst der Verlauf der Erkrankung zeigt, ob die Einschätzung zum Therapiezeitpunkt die Richtige war.

Es bleibt nicht zuletzt zu erwähnen, dass in den letzten 10 Jahren sowohl für GIST als auch für gastrointestinale NET innovative Entwicklungen der Nuklearmedizin zur Diagnostik,
Therapie und Therapieevaluation erfolgreich in den Klinikalltag Einzug hielten [6, 9].

Neben grundlegenden Unterschieden in Diagnostik und Therapie von gastrointestinalen NET und GIST verlangt die Differenziertheit und Komplexität bei Diagnostik und Therapie dieser Erkrankungen nach einem besonders hohen Maß an interdisziplinärer Zusammenarbeit. Das seltene Auftreten beider Tumorgruppen erhöht diesen Anspruch und war Inspiration, dieses Themenheft zusammenzustellen.

\section{Literatur}

1 Wardelmann E: Molekularbiologie und Prognosefaktoren gastrointestinaler mesenchymaler Tumoren. Chir Gastroenterol 2007;23(2):113-118.

2 Neid M, Wittekind C: Epidemiologie, Pathologie, Stadieneinteilung gastrointestinaler mesenchymaler und endokriner Tumoren. Chir Gastroenterol 2007;23(2):108-112.

$\checkmark 3$ Reichardt P: Medikamentöse Therapiemöglichkeiten und Ergebnisse bei gastrointestinalen mesenchymalen Tumoren. Chir Gastroenterol 2007;23(2): 133-136.
4 Würl P: Möglichkeiten der operativen Therapie fortgeschrittener und rezidivierender intraabdomineller und retroperitonealer Sarkome. Chir Gastroenterol 2007;23(2):137-144.

5 Vogelsang H: Neuroendokrine Tumoren des gastroenteropankreatischen Systems: Von multidisziplinärer Vielfalt zur interdisziplinären Einheit eine Standortbestimmung. Chir Gastroenterol 2007;23(2):145-150

6 Busse FP, Mössner J, Stumvoll M, Kluge R, Schoppmeyer K: Nichtoperative Therapiemöglichkeiten gastroenteropankreatischer neuroendokriner Tumoren. Chir Gastroenterol 2007;23(2):158165 .
7 Strate T, Schurr P, Izbicki JR: Reklassifizierung der neuroendokrinen Pankreastumoren und deren Auswirkung auf die operative und präoperative Strategie. Chir Gastroenterol 2007;23(2):151-157.

8 Hoffmann JN, Jauch KW: Chirurgische Therapie neuroendokriner nicht pankreatischer Tumoren des Gastrointestinaltrakts. Chir Gastroenterol 2007;23(2):127-132.

9 Gellißen J, Kapsimalakou S, Begum N, Krüger A, Stoeckelhuber BM, Lubienski A, Helmberger T: Bildgebungsverfahren bei neuroendokrinen und mesenchymalen Tumoren des Gastrointestinaltrakts. Chir Gastroenterol 2007;23(2):119-126. 